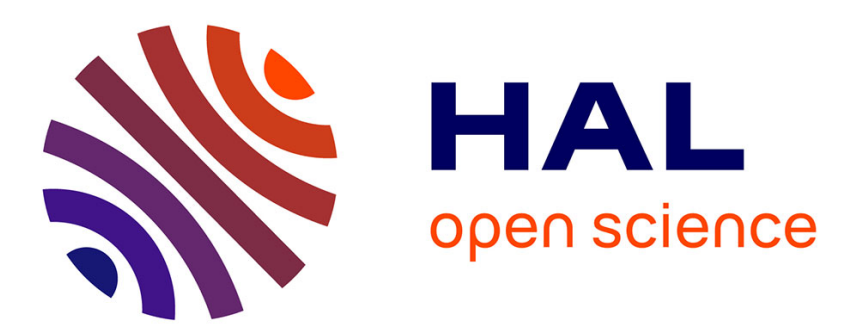

\title{
A constitutive relation error estimator based on traction-free recovery of the equilibrated stress
}

L. Gallimard

\section{To cite this version:}

L. Gallimard. A constitutive relation error estimator based on traction-free recovery of the equilibrated stress. International Journal for Numerical Methods in Engineering, 2009, 78 (4), pp.460 - 482. 10.1002/nme.2496 . hal-01689801

\section{HAL Id: hal-01689801 \\ https://hal.parisnanterre.fr/hal-01689801}

Submitted on 22 Jan 2018

HAL is a multi-disciplinary open access archive for the deposit and dissemination of scientific research documents, whether they are published or not. The documents may come from teaching and research institutions in France or abroad, or from public or private research centers.
L'archive ouverte pluridisciplinaire HAL, est destinée au dépôt et à la diffusion de documents scientifiques de niveau recherche, publiés ou non, émanant des établissements d'enseignement et de recherche français ou étrangers, des laboratoires publics ou privés. 


\title{
A constitutive relation error estimator based on traction-free recovery of the equilibrated stress
}

\author{
L. Gallimard*,† \\ LEME, Université Paris 10, 50 rue de Sèvres-92410 Ville d'Avray, France
}

\begin{abstract}
SUMMARY
A new methodology for recovering equilibrated stress fields is presented, which is based on traction-free subdomains' computations. It allows a rather simple implementation in a standard finite element code compared with the standard technique for recovering equilibrated tractions. These equilibrated stresses are used to compute a constitutive relation error estimator for a finite element model in 2D linear elasticity. A lower bound and an upper bound for the discretization error are derived from the error in the constitutive relation. These bounds in the discretization error are used to build lower and upper bounds for local quantities of interest. Copyright (C) 2008 John Wiley \& Sons, Ltd.
\end{abstract}

KEY WORDS: finite element method; equilibrated stress recovery; constitutive relation error; error bounds; goal-oriented error estimation

\section{INTRODUCTION}

The finite element (FE) discretization of a continuous mechanical model leads to a partial loss of the information contained in the continuous model and, thus, to the introduction of discretization errors. Methods have been developed over many years to evaluate the global quality of FE analyses [1-3]. For linear problems, all these methods provide a global energy-based estimate of the discretization error. Most of the time, such global information is insufficient for dimensioning purposes in mechanical design. In many common situations, the dimensioning criteria involve local quantities (stresses, displacements, intensity factors, etc.). The development of error estimators for such quantities was initiated in the 80s [4,5]. Recently, numerous works have been published which provide error estimates and bounds for several local quantities of interest [6-12]. In these

\footnotetext{
*Correspondence to: L. Gallimard, LEME, Université Paris 10, 50 rue de Sèvres-92410 Ville d'Avray, France.

${ }^{\dagger}$ E-mail: laurent.gallimard@u-paris10.fr 
works, the bounds for the quantities of interest are evaluated starting from the computation of an estimation of a global energy error.

As far as we know the error estimators based on the recovery of equilibrated stress (or equilibrated residuals) are the only type of estimators ensuring easily computable bounds for the error in linear analysis $[2,10,12-18]$. In this paper we consider the approach based on the concept of constitutive relation error (CRE) introduced in [2]. One difficulty of this approach is the construction of equilibrated stress fields from the FE solution and the data. The classical technique of construction proposed [13], which requires equilibrated traction recovery, is difficult to introduce in a standard FE code because the tractions are expressed on the edges of the elements of the mesh.

This paper proposes a new technique to recover equilibrated stress fields, which is easier to introduce in an FE code. This technique is an extension of the CRE estimators in the work of Machiels et al. [14] on residual error estimators. We propose a new lower bound for the error in the constitutive relation, as well.

The paper is organized as follows: In Section 2, we briefly recall the basics of constitutive relation error estimators in linear elasticity. In Section 3 we introduce a new technique to build equilibrated stress fields. Then, in Section 4, we introduce the approach for obtaining a lower bound of the energy norm. In Section 5, a procedure to build the bounds for a quantity of interest is presented. Finally, numerical examples illustrating the behavior of the global and local bounds are presented in Section 6.

\section{THE ERROR IN CONSTITUTIVE RELATION}

\subsection{The problem to be solved}

Let us consider a $2 \mathrm{D}$ elastic structure defined in a domain $\Omega$ bounded by $\partial \Omega$. The external actions on the structure are represented by a prescribed displacement $\mathbf{u}_{\mathrm{d}}$ over a subset $\partial_{1} \Omega$ of the boundary, a surface force density $\mathbf{T}$ defined over $\partial_{2} \Omega=\partial \Omega-\partial_{1} \Omega$, and a body force density $\mathbf{b}$ defined in $\Omega$. Hooke's operator of the material is denoted by $\mathbf{K}$. Thus, the problem can be formulated as: Find a displacement field $\mathbf{u}$ and a stress field $\boldsymbol{\sigma}$ defined in $\Omega$ which verify

\section{- the kinematic constraints:}

$$
\mathbf{u} \in \mathscr{U} \quad \text { and }\left.\quad \mathbf{u}\right|_{\partial_{1} \Omega}=\mathbf{u}_{\mathrm{d}}
$$

- the equilibrium equations:

$$
\boldsymbol{\sigma} \in \mathscr{S} \quad \text { and } \quad \forall \mathbf{u}^{*} \in \mathscr{U}_{0} \quad-\int_{\Omega} \boldsymbol{\sigma}: \varepsilon\left(\mathbf{u}^{*}\right) \mathrm{d} \Omega+\int_{\Omega} \mathbf{b} \cdot \mathbf{u}^{*} \mathrm{~d} \Omega+\int_{\partial_{2} \Omega} \mathbf{T} \cdot \mathbf{u}^{*} \mathrm{~d} \Gamma=0
$$

- the constitutive relation:

$$
\boldsymbol{\sigma}=\mathbf{K} \varepsilon(\mathbf{u})
$$

$\mathscr{U}$ is the space in which the displacement field is being sought, $\mathscr{S}=\mathscr{L}_{2}[\Omega]^{3}$ the space of the stresses, $\mathscr{U}_{0}$ the space of the fields in $\mathscr{U}$ which are zero on $\partial_{1} \Omega$, and $\varepsilon(\mathbf{u})$ denotes the linearized deformation associated with the displacement

$$
[\varepsilon(\mathbf{u})]_{i j}=\frac{1}{2}\left(u_{i, j}+u_{j, i}\right)
$$


The kinematic's constraints are taken homogeneous in the developments for the sake of simplicity (i.e. $\mathbf{u}_{\mathrm{d}}=\mathbf{0}$ ). The weak form of the problem reads: find $\mathbf{u} \in \mathscr{U}_{0}$ such that

$$
-\int_{\Omega} \mathbf{K} \varepsilon(\mathbf{u}): \varepsilon\left(\mathbf{u}^{*}\right) \mathrm{d} \Omega+\int_{\Omega} \mathbf{b} \cdot \mathbf{u}^{*} \mathrm{~d} \Omega+\int_{\partial_{2} \Omega} \mathbf{T} \cdot \mathbf{u}^{*} \mathrm{~d} \Gamma=0 \quad \forall \mathbf{u}^{*} \in \mathscr{U}_{0}
$$

The Galerkin FE method provides an approximation $\mathbf{u}_{h}$ to $\mathbf{u}$ in an FE space $\mathscr{U}^{h} \subset \mathscr{U}$. The finite-dimension space $\mathscr{U}^{h}$ is associated with an FE mesh of characteristic size $h$. Let $\mathscr{P}_{h}$ denote a partition of $\Omega$ into elements $E_{k}$. This partition is assumed to verify $\Omega=\bigcup_{k} E_{k}$ (i.e. the mesh cover the whole domain) with $E_{i} \cap E_{j}=\emptyset$ for any $i$ different from $j$. The discretized problem is: find $\mathbf{u}_{h} \in \mathscr{U}_{0}^{h}$ such that

$$
-\int_{\Omega} \mathbf{K} \varepsilon\left(\mathbf{u}_{h}\right): \varepsilon\left(\mathbf{u}_{h}^{*}\right) \mathrm{d} \Omega+\int_{\Omega} \mathbf{b} \cdot \mathbf{u}_{h}^{*} \mathrm{~d} \Omega+\int_{\partial_{2} \Omega} \mathbf{T} \cdot \mathbf{u}_{h}^{*} \mathrm{~d} \Gamma=0 \quad \forall \mathbf{u}_{h}^{*} \in \mathscr{U}_{0}^{h}
$$

where $\mathscr{U}_{0}^{h}=\left\{\mathbf{u}_{h} \in \mathscr{U}^{h} ;\left.\mathbf{u}_{h}\right|_{\partial_{1} \Omega}=\mathbf{0}\right\}$.

The corresponding stress field is calculated using the constitutive relation

$$
\boldsymbol{\sigma}_{h}=\mathbf{K} \varepsilon\left(\mathbf{u}_{h}\right)
$$

The discretization error $\mathbf{e}_{h}$ is the difference between the FE solution for the displacement and the actual solution of the problem defined by Equation (5)

$$
\mathbf{e}_{h}=\mathbf{u}-\mathbf{u}_{h}
$$

Traditionally, the energy norm is used as the measure of the error

$$
e_{h}=\left\|\mathbf{u}-\mathbf{u}_{h}\right\|_{u}=\left\|\boldsymbol{\sigma}-\boldsymbol{\sigma}_{h}\right\|_{\sigma}
$$

where

$$
\|\mathbf{u}\|_{u}=\left[\int_{\Omega} \varepsilon(\mathbf{u}): \mathbf{K} \varepsilon(\mathbf{u}) \mathrm{d} \Omega\right]^{1 / 2} \quad \text { and } \quad\|\boldsymbol{\sigma}\|_{\sigma}=\left[\int_{\Omega} \boldsymbol{\sigma}: \mathbf{K}^{-1} \boldsymbol{\sigma} \mathrm{d} \Omega\right]^{1 / 2}
$$

\subsection{Definition of the CRE}

The approach based on the CRE relies on a partition of the equations of the problem to be solved into two groups [2]. In linear elasticity, the first group consists of the kinematic constraints (1) and the equilibrium equations (2); the constitutive relation (3) constitutes the second group. Let us consider an approximate solution of the problem, denoted by $(\hat{\mathbf{u}}, \hat{\boldsymbol{\sigma}})$, which verifies the first group of equations:

- the field $\hat{\mathbf{u}}$ verifies (1) (i.e. $\hat{\mathbf{u}}$ is kinematically admissible),

- the field $\hat{\boldsymbol{\sigma}}$ verifies (2) (i.e. $\hat{\boldsymbol{\sigma}}$ is statically admissible).

The fields $(\hat{\mathbf{u}}, \hat{\boldsymbol{\sigma}})$ are said to be an admissible solution. If $(\hat{\mathbf{u}}, \hat{\boldsymbol{\sigma}})$ verifies the constitutive relation (3) in $\Omega$, then $(\hat{\mathbf{u}}, \hat{\boldsymbol{\sigma}})=(\mathbf{u}, \boldsymbol{\sigma})$ (i.e. the exact solution is found). If, however, $(\hat{\mathbf{u}}, \hat{\boldsymbol{\sigma}})$ does not verify the constitutive relation, the quality of this admissible solution is measured by the CRE error $\hat{e}(\hat{\mathbf{u}}, \hat{\boldsymbol{\sigma}})$, which is defined with respect to the constitutive relation

$$
\hat{e}(\hat{\mathbf{u}}, \hat{\boldsymbol{\sigma}})=\left[\int_{\Omega}(\hat{\boldsymbol{\sigma}}-\mathbf{K} \varepsilon(\hat{\mathbf{u}})): \mathbf{K}^{-1}(\hat{\boldsymbol{\sigma}}-\mathbf{K} \varepsilon(\hat{\mathbf{u}})) \mathrm{d} \Omega\right]^{1 / 2}=\|\hat{\boldsymbol{\sigma}}-\mathbf{K} \varepsilon(\hat{\mathbf{u}})\|_{\sigma}
$$


A relative error $\hat{\varepsilon}$ is defined by

$$
\hat{\varepsilon}(\hat{\mathbf{u}}, \hat{\boldsymbol{\sigma}})=\frac{\hat{e}(\hat{\mathbf{u}}, \hat{\boldsymbol{\sigma}})}{\left[\int_{\Omega} \hat{\boldsymbol{\sigma}}^{*} \mathbf{K}^{-1} \hat{\boldsymbol{\sigma}}^{*} \mathrm{~d} \Omega\right]^{1 / 2}}
$$

with

$$
\hat{\boldsymbol{\sigma}}^{*}=\frac{1}{2}(\hat{\boldsymbol{\sigma}}+\mathbf{K} \varepsilon(\hat{\mathbf{u}}))
$$

\subsection{Application of the CRE to FE computations}

A key point to develop a CRE estimator is the construction of an admissible solution $\left(\hat{\mathbf{u}}_{h}, \hat{\boldsymbol{\sigma}}_{h}\right)$ from the FE solution $\left(\mathbf{u}_{h}, \boldsymbol{\sigma}_{h}\right)$ and the data.

- Since the FE displacement field verifies the kinematic constraints, one takes

$$
\hat{\mathbf{u}}_{h}=\mathbf{u}_{h} \quad \text { in } \Omega
$$

- However, the stress field $\boldsymbol{\sigma}_{h}$ does not verify the equilibrium equations (2). A method to recover a statically admissible stress field $\hat{\boldsymbol{\sigma}}_{h}$ from $\boldsymbol{\sigma}_{h}$ and the data have been under development for several years $[9,13,19,20]$. The main features of the recovery method are described in Section 3.1 .

The CRE associated with the admissible solution $\left(\mathbf{u}_{h}, \hat{\boldsymbol{\sigma}}_{h}\right)$ is denoted as $\hat{e}\left(\mathbf{u}_{h}, \hat{\boldsymbol{\sigma}}_{h}\right)$

$$
\hat{e}\left(\mathbf{u}_{h}, \hat{\boldsymbol{\sigma}}_{h}\right)=\left[\int_{\Omega}\left(\hat{\boldsymbol{\sigma}}_{h}-\mathbf{K} \varepsilon\left(\mathbf{u}_{h}\right)\right): \mathbf{K}^{-1}\left(\hat{\boldsymbol{\sigma}}_{h}-\mathbf{K} \varepsilon\left(\mathbf{u}_{h}\right)\right) \mathrm{d} \Omega\right]^{1 / 2}=\left\|\hat{\boldsymbol{\sigma}}_{h}-\mathbf{K} \varepsilon\left(\mathbf{u}_{h}\right)\right\|_{\sigma}
$$

\subsection{Upper bound estimate of the discretization error in the energy norm}

An essential property is that the CRE estimator $\hat{e}\left(\mathbf{u}_{h}, \hat{\boldsymbol{\sigma}}_{h}\right)$ is an upper bound of the discretization error measure $e_{h}$. The Prager-Synge theorem [21] leads to the following inequality:

$$
e_{h} \leqslant \hat{e}\left(\mathbf{u}_{h}, \hat{\boldsymbol{\sigma}}_{h}\right)
$$

The proof of this property is easily obtained by introducing the exact solution $\boldsymbol{\sigma}=\mathbf{K} \varepsilon(\mathbf{u})$ in the expression of the CRE estimator

$$
\begin{aligned}
\hat{e}\left(\mathbf{u}_{h}, \hat{\boldsymbol{\sigma}}_{h}\right)^{2} & =\left\|\hat{\boldsymbol{\sigma}}_{h}-\mathbf{K} \varepsilon\left(\mathbf{u}_{h}\right)\right\|_{\sigma}^{2} \\
& =\left\|\hat{\boldsymbol{\sigma}}_{h}-\boldsymbol{\sigma}+\mathbf{K} \varepsilon(\mathbf{u})-\mathbf{K} \varepsilon\left(\mathbf{u}_{h}\right)\right\|_{\sigma}^{2} \\
& =\left\|\hat{\boldsymbol{\sigma}}_{h}-\boldsymbol{\sigma}\right\|_{\sigma}^{2}+\left\|\mathbf{K} \varepsilon(\mathbf{u})-\mathbf{K} \varepsilon\left(\mathbf{u}_{h}\right)\right\|_{\sigma}^{2}+2 \int_{\Omega}\left(\hat{\boldsymbol{\sigma}}_{h}-\boldsymbol{\sigma}\right): \varepsilon\left(\mathbf{u}-\mathbf{u}_{h}\right) \mathrm{d} \Omega \\
& =\left\|\hat{\boldsymbol{\sigma}}_{h}-\boldsymbol{\sigma}\right\|_{\sigma}^{2}+\left\|\mathbf{u}-\mathbf{u}_{h}\right\|_{u}^{2}+2 \int_{\Omega}\left(\hat{\boldsymbol{\sigma}}_{h}-\boldsymbol{\sigma}\right): \varepsilon\left(\mathbf{e}_{h}\right) \mathrm{d} \Omega
\end{aligned}
$$

As $\hat{\boldsymbol{\sigma}}_{h}$ is an equilibrated stress, and $\mathbf{e}_{h}$ belongs to $\mathscr{U}_{0}$, the following relation holds:

$$
\int_{\Omega} \hat{\boldsymbol{\sigma}}_{h}: \varepsilon\left(\mathbf{e}_{h}\right) \mathrm{d} \Omega=\int_{\Omega} \boldsymbol{\sigma}: \varepsilon\left(\mathbf{e}_{h}\right) \mathrm{d} \Omega
$$


and hence

$$
\int_{\Omega}\left(\hat{\boldsymbol{\sigma}}_{h}-\boldsymbol{\sigma}\right): \varepsilon\left(\mathbf{e}_{h}\right) \mathrm{d} \Omega=0
$$

The proof is completed by introducing Equation (13) and the definition of discretization error in the energy norm in Equation (12)

$$
\hat{e}\left(\mathbf{u}_{h}, \hat{\boldsymbol{\sigma}}_{h}\right)^{2}=\left\|\hat{\boldsymbol{\sigma}}_{h}-\boldsymbol{\sigma}\right\|_{\sigma}^{2}+e_{h}^{2}
$$

\section{RECOVERY OF AN EQUILIBRATED STRESS FIELD}

\subsection{Standard method}

This method has been first introduced in [2], and further developed in several papers $[9,13,19,20]$. It is based on a prolongation condition used to link $\hat{\boldsymbol{\sigma}}_{h}$ to the FE stress $\boldsymbol{\sigma}_{h}$

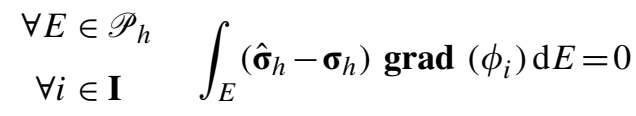

where $E$ denotes an arbitrary element, $\mathbf{I}$ is the set of the mesh nodes, and $\phi_{i}$ the FE scalar shape function associated with the node $i$. It involves two steps:

- The first step consists of constructing, on the element edges, surface force densities $\hat{\mathbf{T}}_{h}$ that represent the vector fields $\hat{\boldsymbol{\sigma}}_{h} \mathbf{n}_{E}$ as

$$
\left[\hat{\boldsymbol{\sigma}}_{h} \mathbf{n}_{E}\right]_{\mid \Gamma}=\eta_{E} \hat{\mathbf{T}}_{h} \quad \text { with } \Gamma \in \partial E
$$

where $\eta_{E}$ is a function that is constant on each edge and whose value is either 1 or -1 , so that on the common edge of two adjacent elements $E$ and $E^{\prime}: \eta_{E}+\eta_{E^{\prime}}=0$. Moreover, these force densities are generated in such a way that the volume load $\mathbf{b}$ and the surface loads $\eta_{E} \hat{\mathbf{T}}_{h}$ are in equilibrium on each element $E$ of the mesh. More details of this procedure can be found in [13].

- The second step consists of constructing on each element $E$ a stress field $\hat{\boldsymbol{\sigma}}_{E}=\hat{\boldsymbol{\sigma}}_{h} \mid E$ solution of the equilibrium equations:

$$
\begin{aligned}
\operatorname{div} \hat{\boldsymbol{\sigma}}_{E}+\mathbf{b} & =0 \quad \text { in } E \\
\hat{\boldsymbol{\sigma}}_{E} \mathbf{n}_{E} & =\eta_{E} \hat{\mathbf{T}}_{h} \quad \text { on } \partial E
\end{aligned}
$$

For a given set of surface force densities, among all the solutions of (15) the best field $\hat{\boldsymbol{\sigma}}_{E}$ is the one that is the solution to the minimization problem:

$$
\min _{\hat{\boldsymbol{\sigma}}_{E} \text { verifying(15) }} \frac{1}{2} \int_{E}\left(\hat{\boldsymbol{\sigma}}_{E}-\boldsymbol{\sigma}_{h}\right): \mathbf{K}^{-1}\left(\hat{\boldsymbol{\sigma}}_{E}-\boldsymbol{\sigma}_{h}\right) \mathrm{d} V
$$

By duality, this is equivalent to seeking a displacement field $\mathbf{u}_{E} \in \mathscr{U}(E)$ such that

$$
\forall \mathbf{u}^{*} \in \mathscr{U}(E) \quad \int_{E} \mathbf{K} \varepsilon\left(\mathbf{u}_{E}\right): \varepsilon\left(\mathbf{u}^{*}\right) \mathrm{d} E-\int_{E} \mathbf{b u} \mathbf{u}^{*} \mathrm{~d} E-\int_{\partial E} \eta_{E} \hat{\mathbf{T}}_{h} \mathbf{u}^{*} \mathrm{~d} \Gamma=0
$$


where $\mathscr{U}(E)$ designates the space of the restriction to $E$ of the fields in $\mathscr{U}$. The stress field $\hat{\boldsymbol{\sigma}}_{E}$ is given by

$$
\hat{\boldsymbol{\sigma}}_{E}=\mathbf{K} \varepsilon\left(\mathbf{u}_{E}\right)
$$

Thus, one can obtain an approximation of $\hat{\boldsymbol{\sigma}}_{E}$ by solving the problem (16) by a classical FE method on $E$. In practice, it is sufficient, in order to obtain a good approximation, to consider either a discretization of $E$ with a single element but an interpolation of degree $p+k$, where $p$ is the degree of the interpolation used in the FE method and $k$ a positive integer, or a subdivision of the element $E$ along with an interpolation of degree $p$. A complete study can be found in [22]. However, provided the loading is sufficiently smooth, it is possible to recover rigorously equilibrated stresses $[13,18]$.

The main drawback of this technique is to require, for the construction of the surface force densities $\hat{T}_{h}$, a data structure that is not natural in a classical FE code (i.e. nodes and integration points defined on the edges of the elements). In the next subsection we present a method to build an equilibrated stress field that requires only the knowledge of the classical integration points and the nodes of the mesh.

\subsection{Method based on traction-free recovery}

This method is based on the partition of unity and uses local subdomains different from the mesh elements. It has been used for the residual error estimators [14, 17, 23, 24]. The principles of this method, associated with a new prolongation condition, can be used to recover statically admissible stress fields without computing the surface loads $\eta_{E} \hat{\mathbf{T}}_{h}$ on the edges of the elements of the mesh. Let $\mathbf{I}_{\mathrm{V}}$ be the set of vertices of $\mathscr{P}_{h}$ and $\lambda_{i}$ the corresponding piecewise linear (or bilinear for square elements) shape functions. The support of $\lambda_{i}$ is the set of the mesh elements that contains the vertice $i$ and is denoted by $\omega_{i}$ (see Figure 1). The sum of the functions $\lambda_{i}$ is a partition of unity

$$
\sum_{i \in \mathbf{I}_{\mathrm{v}}} \lambda_{i}=1
$$

Now, the statical stress field $\hat{\boldsymbol{\sigma}}_{h}$ is sought as a sum of stress fields $\hat{\boldsymbol{\sigma}}_{h}^{i}=\lambda_{i} \hat{\boldsymbol{\sigma}}_{h}$ computed in each subdomain $\omega_{i}$ such that $\hat{\boldsymbol{\sigma}}_{h}^{i}$ vanishes in $\Omega-\omega_{i}$. Moreover, $\hat{\boldsymbol{\sigma}}_{h}$ is linked to the FE stress $\boldsymbol{\sigma}_{h}$ by the

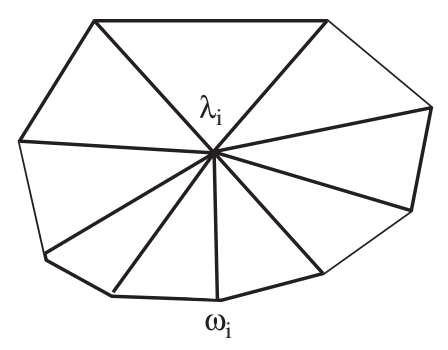

Figure 1. $\omega_{i}$ : support of a linear shape function $\lambda_{i}$. 
following prolongation condition defined in $\omega_{i}$ :

$$
\int_{\omega_{i}}\left(\left(\hat{\boldsymbol{\sigma}}_{h}-\boldsymbol{\sigma}_{h}\right) \operatorname{grad} \lambda_{i}\right) \mathbf{u}^{*} \mathrm{~d} \omega=0 \quad \forall \mathbf{u}^{*} \in \mathscr{U}_{0}\left(\omega_{i}\right)
$$

where $\mathscr{U}_{0}\left(\omega_{i}\right)$ is the local restriction of the set $\mathscr{U}_{0}$ on the support $\omega_{i}$.

Using Equation (17), the following equality holds:

$$
\hat{\boldsymbol{\sigma}}_{h}=\left(\sum_{i \in \mathbf{I}_{\mathrm{v}}} \lambda_{i}\right) \hat{\boldsymbol{\sigma}}_{h}=\sum_{i \in \mathbf{I}_{\mathrm{v}}}\left(\lambda_{i} \hat{\boldsymbol{\sigma}}_{h}\right)=\sum_{i \in \mathbf{I}_{\mathrm{v}}} \hat{\boldsymbol{\sigma}}_{h}^{i}
$$

and $\hat{\boldsymbol{\sigma}}_{h}^{i}$ satisfies the following equilibrium equation in $\omega_{i}$ :

$$
\begin{aligned}
\operatorname{div} \hat{\boldsymbol{\sigma}}_{h}^{i} & =\operatorname{div}\left(\lambda_{i} \hat{\boldsymbol{\sigma}}_{h}\right) \\
& =\lambda_{i} \operatorname{div} \hat{\boldsymbol{\sigma}}_{h}+\hat{\boldsymbol{\sigma}}_{h} \operatorname{grad} \lambda_{i} \\
& =-\lambda_{i} \mathbf{b}+\hat{\boldsymbol{\sigma}}_{h} \operatorname{grad} \lambda_{i}
\end{aligned}
$$

as well as the following boundary conditions on $\partial \omega_{i}$ :

$$
\begin{aligned}
& \hat{\boldsymbol{\sigma}}_{h}^{i} \mathbf{n}=\lambda_{i} \mathbf{T} \quad \text { on } \partial_{2}^{i} \boldsymbol{\Omega} \\
& \hat{\boldsymbol{\sigma}}_{h}^{i} \mathbf{n}=\mathbf{0} \quad \text { on } \partial \omega_{i}-\left(\partial_{1}^{i} \Omega \cup \partial_{2}^{i} \boldsymbol{\Omega}\right)
\end{aligned}
$$

where

$$
\partial_{2}^{i} \Omega=\partial \omega_{i} \cap \partial_{2} \Omega, \quad \partial_{1}^{i} \Omega=\partial \omega_{i} \cap \partial_{1} \Omega
$$

The weak solutions of the problem defined by Equations (19) and (20) are the fields $\hat{\boldsymbol{\sigma}}_{h}^{i}$ verifying

$$
\int_{\omega_{i}} \hat{\boldsymbol{\sigma}}_{h}^{i} \varepsilon\left(\mathbf{u}^{*}\right) \mathrm{d} \omega=\int_{\omega_{i}}\left(\lambda_{i} \mathbf{b}-\hat{\boldsymbol{\sigma}}_{h} \operatorname{grad} \lambda_{i}\right) \mathbf{u}^{*} \mathrm{~d} \omega+\int_{\partial_{2}^{i} \Omega} \lambda_{i} \mathbf{T} \mathbf{u}^{*} \mathrm{~d} \Gamma \quad \forall \mathbf{u}^{*} \in \mathscr{U}_{0}\left(\omega_{i}\right)
$$

Note that if $\partial_{2}^{i} \Omega=\emptyset$ the corresponding term disappears from the equation.

Introducing the prolongation condition (18) in Equation (21), one obtains

$$
\begin{aligned}
\forall \mathbf{u}^{*} \in \mathscr{U}_{0}\left(\omega_{i}\right) \quad \int_{\omega_{i}} \hat{\boldsymbol{\sigma}}_{h}^{i} \varepsilon\left(\mathbf{u}^{*}\right) \mathrm{d} \omega= & \int_{\omega_{i}}\left(\lambda_{i} \mathbf{b}-\boldsymbol{\sigma}_{h} \operatorname{grad} \lambda_{i}\right) \mathbf{u}^{*} \mathrm{~d} \omega+\int_{\partial_{2}^{i} \Omega} \lambda_{i} \mathbf{T} \mathbf{u}^{*} \mathrm{~d} \Gamma \\
= & \int_{\omega_{i}}\left(\lambda_{i} \boldsymbol{\sigma}_{h} \varepsilon\left(\mathbf{u}^{*}\right)-\boldsymbol{\sigma}_{h}: \varepsilon\left(\lambda_{i} \mathbf{u}^{*}\right)\right) \mathrm{d} \omega \\
& +\int_{\omega_{i}} \mathbf{b}\left(\lambda_{i} \mathbf{u}^{*}\right) \mathrm{d} \omega+\int_{\partial_{2}^{i} \Omega} \mathbf{T}\left(\lambda_{i} \mathbf{u}^{*}\right) \mathrm{d} \Gamma
\end{aligned}
$$


Equation (22) is solvable on $\omega_{i}$ if, for any rigid body motion $\mathbf{u}_{\mathrm{rb}}$ of $\omega_{i}$, the right-hand term is equal to zero

$$
-\int_{\omega_{i}} \boldsymbol{\sigma}_{h}: \varepsilon\left(\lambda_{i} \mathbf{u}_{\mathrm{rb}}\right) \mathrm{d} \omega+\int_{\omega_{i}} \mathbf{b}\left(\lambda_{i} \mathbf{u}_{\mathrm{rb}}\right) \mathrm{d} \omega+\int_{\partial_{2}^{i} \Omega} \mathbf{T}\left(\lambda_{i} \mathbf{u}_{\mathrm{rb}}\right) \mathrm{d} \Gamma=0
$$

As $\boldsymbol{\sigma}_{h}$ satisfies the equilibrium equations in the FE sense, Equation (23) is satisfied if $\lambda_{i} \mathbf{u}_{\mathrm{rb}}$ belongs to the FE space $\mathscr{U}_{0}^{h}$. If $\lambda_{i} \mathbf{u}_{\mathrm{rb}}$ does not belong to the FE space $\mathscr{U}_{0}^{h}$ (for instance, for linear triangular elements), it is necessary to add forces $\mathbf{F}_{i}(M)$ to the loading such that the right-hand term is equal to zero for any rigid body motion of $\omega_{i}$ and that $\sum_{i \in \mathbf{I}_{\mathrm{v}}} \mathbf{F}_{i}(M)=\mathbf{0}$. This point will be developed in the Appendix.

\subsection{Practical implementation}

The construction of an admissible stress $\hat{\boldsymbol{\sigma}}_{h}$ requires the resolution of local problems (22). The stress field $\hat{\boldsymbol{\sigma}}_{h}^{i}$ is sought as the sum of the FE stress field and a correction stress field

$$
\hat{\boldsymbol{\sigma}}_{h}^{i}=\lambda_{i} \boldsymbol{\sigma}_{h}+\mathbf{K} \varepsilon\left(\boldsymbol{\delta} \hat{\mathbf{u}}^{i}\right)
$$

Equation (22) becomes: find $\delta \hat{\mathbf{u}}^{i}$ in $\mathscr{U}_{0}\left(\omega_{i}\right)$ such that

$$
\begin{aligned}
\forall \mathbf{u}^{*} \in \mathscr{U}_{0}\left(\omega_{i}\right) \quad \int_{\omega_{i}} \mathbf{K} \varepsilon\left(\boldsymbol{\delta} \hat{\mathbf{u}}^{i}\right): \varepsilon\left(\mathbf{u}^{*}\right) \mathrm{d} \omega= & \int_{\omega_{i}}\left(\lambda_{i} \mathbf{b}-\boldsymbol{\sigma}_{h} \mathbf{g r a d} \lambda_{i}\right) \mathbf{u}^{*} \mathrm{~d} \omega \\
& -\int_{\omega_{i}} \lambda_{i} \boldsymbol{\sigma}_{h} \varepsilon\left(\mathbf{u}^{*}\right) \mathrm{d} \omega+\int_{\hat{\partial}_{2}^{i} \Omega} \lambda_{i} \mathbf{T} \mathbf{u}^{*} \mathrm{~d} \Gamma
\end{aligned}
$$

In practice the resolution is performed in the same way as problem (16) in the second step of the standard method (see Section 3.1). An approximation of $\delta \hat{\mathbf{u}}^{i}$ is computed by solving the problem (24) by a classical FE method on $\omega_{i}$. A subdivision of the initial FE mesh on $\omega_{i}$ is introduced: $\mathscr{P}_{h, S}\left(\omega_{i}\right)$ where $S$ is the subdivision per edge in all directions. Let $\mathscr{U}^{h, S}\left(\omega_{i}\right)$ be the associated FE space, $\mathscr{U}_{0}^{h, S}\left(\omega_{i}\right)$ the space of the fields in $\mathscr{U}^{h, S}\left(\omega_{i}\right)$ which are equal to 0 on $\partial_{1} \Omega$. The problem (24) becomes: find $\delta \hat{\mathbf{u}}^{i} \in \mathscr{U}_{0}^{h, S}\left(\omega_{i}\right)$ such that

$$
\begin{aligned}
\forall \mathbf{u}^{*} \in \mathscr{U}_{0}^{h, S}\left(\omega_{i}\right) \quad \int_{\omega_{i}} \mathbf{K} \varepsilon\left(\delta \hat{\mathbf{u}}^{i}\right): \varepsilon\left(\mathbf{u}^{*}\right) \mathrm{d} \omega= & \int_{\omega_{i}}\left(\lambda_{i} \mathbf{b}-\boldsymbol{\sigma}_{h} \mathbf{g r a d} \lambda_{i}\right) \mathbf{u}^{*} \mathrm{~d} \omega \\
& -\int_{\omega_{i}} \lambda_{i} \boldsymbol{\sigma}_{h} \varepsilon\left(\mathbf{u}^{*}\right)+\int_{\partial_{2}^{i} \Omega} \lambda_{i} \mathbf{T} \mathbf{u}^{*} \mathrm{~d} \Gamma
\end{aligned}
$$

The main advantage of the traction-free recovery method over the standard method is the simplicity of its implementation. The traction-free error estimator requires only one additional information to the standard data structure of an FE code. This information is the set of the elements that surround a given vertex node, and is easily determined from the connection table. Moreover, it does not require the computation of the flux along the edges of the elements. By comparison the standard method needs a data structure to express the flux on the edges of the elements and a connection between the edges, the elements and the nodes of the mesh, as well as integration points and weight on these edges. Finally, the boundary conditions of the local problems are easy to introduce in the standard structure of an FE code. 


\section{LOWER BOUND}

\subsection{Definition of the lower bound}

Let us consider a displacement field $\tilde{\mathbf{u}}_{h}$ that satisfy the kinematic constraints (Equation (1)). Then, as the admissible stress field built to compute the upper bound $\hat{\boldsymbol{\sigma}}_{h}$ satisfies the equilibrium equation (2)

$$
\int_{\Omega} \hat{\boldsymbol{\sigma}}_{h}: \varepsilon\left(\tilde{\mathbf{u}}_{h}-\mathbf{u}_{h}\right) \mathrm{d} \Omega=\int_{\Omega} \boldsymbol{\sigma}: \varepsilon\left(\tilde{\mathbf{u}}_{h}-\mathbf{u}_{h}\right) \mathrm{d} \Omega
$$

By subtracting on each side of the equation the quantity $\int_{\Omega} \boldsymbol{\sigma}_{h}: \varepsilon\left(\tilde{\mathbf{u}}_{h}-\mathbf{u}_{h}\right) \mathrm{d} \Omega$ and by using the Cauchy-Schwarz inequality, we obtain the following relation:

$$
\left|\int_{\Omega}\left(\hat{\boldsymbol{\sigma}}_{h}-\boldsymbol{\sigma}_{h}\right): \varepsilon\left(\tilde{\mathbf{u}}_{h}-\mathbf{u}_{h}\right) \mathrm{d} \Omega\right| \leqslant\left\|\boldsymbol{\sigma}-\boldsymbol{\sigma}_{h}\right\|_{\sigma}\left\|\tilde{\mathbf{u}}_{h}-\mathbf{u}_{h}\right\|_{u}=e_{h}\left\|\tilde{\mathbf{u}}_{h}-\mathbf{u}_{h}\right\|_{u}
$$

where $\|\cdot\|_{\sigma}$ and $\|\cdot\|_{u}$ are the classical energy norms defined in Equation (10). From Equation (26) a lower bound $\tilde{e}\left(\tilde{\mathbf{u}}_{h}-\mathbf{u}_{h}, \hat{\boldsymbol{\sigma}}_{h}-\boldsymbol{\sigma}_{h}\right)$ of the discretization error $e_{h}$ can be derived

$$
\tilde{e}\left(\tilde{\mathbf{u}}_{h}-\mathbf{u}_{h}, \hat{\boldsymbol{\sigma}}_{h}-\boldsymbol{\sigma}_{h}\right) \leqslant e_{h}
$$

with

$$
\tilde{e}\left(\tilde{\mathbf{u}}_{h}-\mathbf{u}_{h}, \hat{\boldsymbol{\sigma}}_{h}-\boldsymbol{\sigma}_{h}\right)=\frac{\left|\int_{\Omega}\left(\hat{\boldsymbol{\sigma}}_{h}-\boldsymbol{\sigma}_{h}\right): \varepsilon\left(\tilde{\mathbf{u}}_{h}-\mathbf{u}_{h}\right) \mathrm{d} \Omega\right|}{\left\|\tilde{\mathbf{u}}_{h}-\mathbf{u}_{h}\right\|_{u}}
$$

\subsection{Practical construction of the lower bound}

To compute the lower bound, it is sufficient to construct an admissible displacement field $\tilde{\mathbf{u}}_{h}$. This displacement field can be decomposed as the sum of the FE displacement field and a correction displacement field $\delta \tilde{\mathbf{u}}_{h}$, where $\delta \tilde{\mathbf{u}}_{h}$ belongs to $\mathscr{U}_{0}$ :

$$
\tilde{\mathbf{u}}_{h}=\mathbf{u}_{h}+\delta \tilde{\mathbf{u}}_{h}
$$

Following the method proposed in Section 3.2 to compute the admissible stress $\hat{\boldsymbol{\sigma}}_{h}, \delta \tilde{\mathbf{u}}_{h}$ is sought as a sum of displacement fields $\delta \tilde{\mathbf{u}}_{h}^{i}$ computed on each subdomain $\omega_{i}$

$$
\delta \tilde{\mathbf{u}}_{h}=\sum_{i \in \mathbf{I}_{\mathrm{v}}} \delta \tilde{\mathbf{u}}_{h}^{i}
$$

To ensure the continuity of $\delta \tilde{\mathbf{u}}_{h}$, a simple way is to search $\delta \tilde{\mathbf{u}}_{h}^{i}$ such that $\delta \tilde{\mathbf{u}}_{h}^{i}$ is equal to zero on $\partial \omega_{i}$. Then $\delta \tilde{\mathbf{u}}_{h}^{i}$ is built in the same FE space as $\delta \hat{\mathbf{u}}^{i}$, and $\delta \tilde{\mathbf{u}}_{h}^{i}$ is the solution of the following FE problem defined on $\omega_{i}$ : find $\delta \tilde{\mathbf{u}}_{h}^{i} \in \mathscr{U}_{0, \partial \omega_{i}}^{h, S}\left(\omega_{i}\right)$ such that

$$
\forall \mathbf{u}^{*} \in \mathscr{U}_{0, \partial \omega_{i}}^{h, S}\left(\omega_{i}\right) \int_{\omega_{i}} \mathbf{K} \varepsilon\left(\delta \tilde{\mathbf{u}}_{h}^{i}\right): \varepsilon\left(\mathbf{u}^{*}\right) \mathrm{d} \omega=\int_{\omega_{i}}\left(\lambda_{i} \mathbf{b}-\boldsymbol{\sigma}_{h} \operatorname{grad} \lambda_{i}\right) \cdot \mathbf{u}^{*} \mathrm{~d} \omega-\int_{\omega_{i}} \lambda_{i} \boldsymbol{\sigma}_{h}: \varepsilon\left(\mathbf{u}^{*}\right) \mathrm{d} \omega
$$

where $\mathscr{U}_{0, \partial \omega_{i}}^{h, S}\left(\omega_{i}\right)$ is the space fields in $\mathscr{U}_{0}^{h, S}\left(\omega_{i}\right)$, which are equal to zero on $\partial \omega_{i}$. This FE problem is similar to the FE problem solved to compute the stress field $\hat{\boldsymbol{\sigma}}_{h}^{i}(25)$, but the Neumann boundary conditions have been replaced by Dirichlet boundary conditions. 


\section{APPLICATION TO THE COMPUTATION OF A GOAL-ORIENTED ERROR ESTIMATOR}

In this section the global upper and lower bounds in the energy norm (defined, respectively, in Sections 2 and 4) are used to obtain bounds for quantities of interest. We briefly recall the techniques developed in the literature for the case where the quantity of interest is a linear functional $L$ of the displacement. In this case, the objective of the calculation is to assess the quality of $I_{h}=L\left(\mathbf{u}_{h}\right)$ by estimating $\left|I-I_{h}\right|$, where $I=L(\mathbf{u})$ (an example of quantity of interest is proposed in Section 6.2). We refer the reader to References [8-11,25] for a detailed description of this approach and to [26-29] for the extension to non-linear quantities of interest.

Owing to the linearity assumption, one has

$$
I-I_{h}=L(\mathbf{u})-L\left(\mathbf{u}_{h}\right)=L\left(\mathbf{u}-\mathbf{u}_{h}\right)=L\left(\mathbf{e}_{h}\right)
$$

thus, the estimate of $\left|I-I_{h}\right|$ is equivalent to the estimate of $\left|L\left(\mathbf{e}_{h}\right)\right|$.

\subsection{Definition of the auxiliary problem}

Let us consider the following auxiliary problem: find $\mathbf{u}^{\text {aux }} \in \mathscr{U}_{0}$ and $\boldsymbol{\sigma}^{\text {aux }}=\mathbf{K} \varepsilon\left(\mathbf{u}^{\text {aux }}\right)$ such that

$$
\int_{\Omega} \mathbf{K} \varepsilon\left(\mathbf{u}^{*}\right): \varepsilon\left(\mathbf{u}^{\mathrm{aux}}\right) \mathrm{d} \Omega=L\left(\mathbf{u}^{*}\right) \quad \forall \mathbf{u}^{*} \in \mathscr{U}_{0}
$$

Replacing $\mathbf{u}^{*}$ by $\mathbf{e}_{h}$, one gets

$$
L\left(\mathbf{e}_{h}\right)=\int_{\Omega} \mathbf{K} \varepsilon\left(\mathbf{e}_{h}\right): \varepsilon\left(\mathbf{u}^{\text {aux }}\right) \mathrm{d} \Omega
$$

The function $\mathbf{u}^{\text {aux }}$ indicates how the discretization error affects the quantity $L\left(\mathbf{e}_{h}\right)$. If $\mathbf{u}^{\text {aux }}$ could be exactly computed, one could determine $L(\mathbf{u})$ directly from the input data, as from (2), (29) and because $\mathbf{u}^{\text {aux }}$ belongs to $\mathscr{U}_{0}$, one would have

$$
L(\mathbf{u})=\int_{\Omega} \mathbf{b} \cdot \mathbf{u}^{\text {aux }} \mathrm{d} \Omega+\int_{\partial_{2} \Omega} \mathbf{T} \cdot \mathbf{u}^{\text {aux }} \mathrm{d} \Gamma
$$

Unfortunately, the problem for the function $\mathbf{u}^{\text {aux }}$ is as complicated to solve as the reference problem for the solution $\mathbf{u}$. However, we can compute an approximate value for $\mathbf{u}^{\text {aux }}$.

The relation (30) is the starting point of the goal-oriented error estimators developed in [5-10]. The approaches proposed in these papers differ on three points:

- the technique developed to approximate $\mathbf{u}^{\text {aux }}$,

- the technique used to obtain lower and upper bounds of $\left|L\left(\mathbf{e}_{h}\right)\right|$,

- the error estimators used to calculate the bounds.

\subsection{Approximate solution of the auxiliary problem}

Let $\mathbf{u}_{h}^{\text {aux }}$ be the FE approximation of $\mathbf{u}^{\text {aux }}$ defined in the FE space $\mathscr{U}_{0}^{h}$ :

$$
\int_{\Omega} \mathbf{K} \varepsilon\left(\mathbf{u}_{h}^{*}\right): \varepsilon\left(\mathbf{u}_{h}^{\text {aux }}\right) \mathrm{d} \Omega=L\left(\mathbf{u}_{h}^{*}\right) \quad \forall \mathbf{u}_{h}^{*} \in \mathscr{U}_{0}^{h}
$$


Because of the orthogonality property, one has

$$
\int_{\Omega} \mathbf{K} \varepsilon\left(\mathbf{e}_{h}\right): \varepsilon\left(\mathbf{u}_{h}^{*}\right) \mathrm{d} \Omega=0 \quad \forall \mathbf{u}_{h}^{*} \in \mathscr{U}_{0}^{h}
$$

Replacing $\mathbf{u}_{h}^{*}$ by $\mathbf{u}_{h}^{\text {aux }}$ the above relation transforms to

$$
\int_{\Omega} \mathbf{K} \varepsilon\left(\mathbf{e}_{h}\right): \varepsilon\left(\mathbf{u}_{h}^{\text {aux }}\right) \mathrm{d} \Omega=0
$$

By combining (30) and (32), one obtains the following relation for the error estimate:

$$
I-I_{h}=L\left(\mathbf{e}_{h}\right)=\int_{\Omega} \mathbf{K} \varepsilon\left(\mathbf{e}_{h}\right): \varepsilon\left(\mathbf{e}_{h}^{\text {aux }}\right) \mathrm{d} \Omega
$$

where $\mathbf{e}_{h}^{\text {aux }}=\mathbf{u}^{\text {aux }}-\mathbf{u}_{h}^{\text {aux }}$ is the discretization error of the auxiliary problem.

\subsection{Bounds of the quantity of interest}

Following [11], the error in the quantity of interest can be written as

$$
\begin{aligned}
I-I_{h} & =\int_{\Omega} \mathbf{K} \varepsilon\left(\mathbf{e}_{h}\right): \varepsilon\left(\mathbf{e}_{h}^{\mathrm{aux}}\right) \mathrm{d} \Omega \\
& =\frac{1}{4}\left\|s \mathbf{e}_{h}+s^{-1} \mathbf{e}_{h}^{\mathrm{aux}}\right\|_{u}^{2}-\frac{1}{4}\left\|s \mathbf{e}_{h}-s^{-1} \mathbf{e}_{h}^{\mathrm{aux}}\right\|_{u}^{2}
\end{aligned}
$$

where $s$ is a scaling factor. The value of $s$ is chosen so that $\left\|s \mathbf{e}_{h}\right\|_{u}$ and $\left\|s^{-1} \mathbf{e}_{h}^{\text {aux }}\right\|_{u}$ have the same amplitude. This implies that

$$
s=\sqrt{\left\|\mathbf{e}_{h}^{\mathrm{aux}}\right\|_{u} /\left\|\mathbf{e}_{h}\right\|_{u}}
$$

However, in practice, the discretization errors $\left\|\mathbf{e}_{h}^{\text {aux }}\right\|_{u}$ and $\left\|\mathbf{e}_{h}\right\|_{u}$ will be replaced by the computed error estimators in the constitutive relation.

Replacing $\mathbf{e}_{h}$ by $\left(\mathbf{u}-\mathbf{u}_{h}\right)$ and $\mathbf{e}_{h}^{\text {aux }}$ by $\left(\mathbf{u}^{\text {aux }}-\mathbf{u}_{h}^{\text {aux }}\right)$ in Equation (34) one easily obtains

$$
I-I_{h}=\frac{1}{4}\left\|\mathbf{u}^{+}-\mathbf{u}_{h}^{+}\right\|_{u}^{2}-\frac{1}{4}\left\|\mathbf{u}^{-}-\mathbf{u}_{h}^{-}\right\|_{u}^{2}
$$

where

$$
\mathbf{u}^{ \pm}=s \mathbf{u} \pm s^{-1} \mathbf{u}^{\text {aux }} \quad \text { and } \quad \mathbf{u}_{h}^{ \pm}=s \mathbf{u}_{h} \pm s^{-1} \mathbf{u}_{h}^{\text {aux }}
$$

In order to determine bounds for the error in the quantity of interest, the error estimates of both the initial and the auxiliary problem are necessary. The methodology proposed in Sections 3 and 4 can be used to build a statically admissible stress field $\hat{\boldsymbol{\sigma}}_{h}^{\text {aux }}$ and a kinematically admissible displacement field $\tilde{\mathbf{u}}_{h}^{\text {aux }}$ for the auxiliary problem. Taking advantage of the linearity of the problems, and introducing the global lower and upper bounds defined, respectively, in Equations (27) and (11), bounds for $\left\|\mathbf{u}^{+}-\mathbf{u}_{h}^{+}\right\|_{u}$ and $\left\|\mathbf{u}^{-}-\mathbf{u}_{h}^{-}\right\|_{u}$ can be built

$$
\begin{aligned}
& \tilde{e}\left(\tilde{\mathbf{u}}_{h}^{+}-\mathbf{u}_{h}^{+}, \hat{\boldsymbol{\sigma}}_{h}^{+}-\boldsymbol{\sigma}_{h}^{+}\right) \leqslant\left\|\mathbf{u}^{+}-\mathbf{u}_{h}^{+}\right\|_{u} \leqslant \hat{e}\left(\mathbf{u}_{h}^{+}, \hat{\boldsymbol{\sigma}}_{h}^{+}\right) \\
& \tilde{e}\left(\tilde{\mathbf{u}}_{h}^{-}-\mathbf{u}_{h}^{-}, \hat{\boldsymbol{\sigma}}_{h}^{-}-\boldsymbol{\sigma}_{h}^{-}\right) \leqslant\left\|\mathbf{u}^{-}-\mathbf{u}_{h}^{-}\right\|_{u} \leqslant \hat{e}\left(\mathbf{u}_{h}^{-}, \hat{\boldsymbol{\sigma}}_{h}^{-}\right)
\end{aligned}
$$


where

$$
\begin{array}{lll}
\hat{\boldsymbol{\sigma}}_{h}^{+}=s \hat{\boldsymbol{\sigma}}_{h}+s^{-1} \hat{\boldsymbol{\sigma}}_{h}^{\text {aux }} & \text { and } & \hat{\boldsymbol{\sigma}}_{h}^{-}=s \hat{\boldsymbol{\sigma}}_{h}-s^{-1} \hat{\boldsymbol{\sigma}}_{h}^{\text {aux }} \\
\tilde{\mathbf{u}}_{h}^{+}=s \tilde{\mathbf{u}}_{h}+s^{-1} \tilde{\mathbf{u}}_{h}^{\text {aux }} & \text { and } & \tilde{\mathbf{u}}_{h}^{-}=s \tilde{\mathbf{u}}_{h}-s^{-1} \tilde{\mathbf{u}}_{h}^{\text {aux }}
\end{array}
$$

From Equations (34) and (36), the classical upper and lower bounds are derived [11]

$$
\eta_{\text {low }} \leqslant I-I_{h} \leqslant \eta_{\text {upp }}
$$

where

$$
\begin{aligned}
& \eta_{\text {low }}=\frac{1}{4} \tilde{e}\left(\tilde{\mathbf{u}}_{h}^{+}-\mathbf{u}_{h}^{+}, \hat{\boldsymbol{\sigma}}_{h}^{+}-\boldsymbol{\sigma}_{h}^{+}\right)^{2}-\frac{1}{4} \hat{e}\left(\mathbf{u}_{h}^{-}, \hat{\boldsymbol{\sigma}}_{h}^{-}\right)^{2} \\
& \eta_{\text {upp }}=\frac{1}{4} \hat{e}\left(\mathbf{u}_{h}^{+}, \hat{\boldsymbol{\sigma}}_{h}^{+}\right)^{2}-\frac{1}{4} \tilde{e}\left(\tilde{\mathbf{u}}_{h}^{-}-\mathbf{u}_{h}^{-}, \hat{\boldsymbol{\sigma}}_{h}^{-}-\boldsymbol{\sigma}_{h}^{-}\right)^{2}
\end{aligned}
$$

The bounds for the quantity of interest $I$ are easily derived from (37)

$$
I_{h}^{\text {upp }}=I_{h}+\eta_{\text {upp }} \quad \text { and } \quad I_{h}^{\text {low }}=I_{h}+\eta_{\text {low }}
$$

The introduction of lower bounds in the energy $\tilde{e}\left(\tilde{\mathbf{u}}_{h}^{+}-\mathbf{u}_{h}^{+}, \hat{\boldsymbol{\sigma}}_{h}^{+}-\boldsymbol{\sigma}_{h}^{+}\right)$and $\tilde{e}\left(\tilde{\mathbf{u}}_{h}^{-}-\mathbf{u}_{h}^{-}, \hat{\boldsymbol{\sigma}}_{h}^{-}-\boldsymbol{\sigma}_{h}^{-}\right)$ leads to much sharper bounds than the classical approach based on the error estimator in the constitutive relation, where the 'local' lower and upper bounds are constructed with only the CRE estimator computed on the initial and auxiliary problem.

$$
\begin{aligned}
& \eta_{\mathrm{low}}^{\mathrm{CRE}}=-\frac{1}{4} \hat{e}\left(\mathbf{u}_{h}^{-}, \hat{\boldsymbol{\sigma}}_{h}^{-}\right)^{2} \\
& \eta_{\mathrm{upp}}^{\mathrm{CRE}}=\frac{1}{4} \hat{e}\left(\mathbf{u}_{h}^{+}, \hat{\boldsymbol{\sigma}}_{h}^{+}\right)^{2}
\end{aligned}
$$

In this case, the bounds for the quantity of interest $I$ are

$$
I_{h}^{\mathrm{upp}, \mathrm{CRE}}=I_{h}+\eta_{\mathrm{upp}}^{\mathrm{CRE}} \quad \text { and } \quad I_{h}^{\mathrm{low}, \mathrm{CRE}}=I_{h}+\eta_{\mathrm{low}}^{\mathrm{CRE}}
$$

\section{NUMERICAL EXAMPLES}

This section is devoted to the study of the error estimates and bounds presented above. In the first example, we analyze the behavior of the global lower and upper bounds in the energy norm on a plate with two holes. The next examples deal with the study of the goal-oriented error estimation for a crack opening problem. In all the cases studied the exact solutions are unknown. The estimates and the error bounds are then verified by comparing results with solutions obtained on fine meshes, which are believed to provide accurate solutions. The quality of the global CRE estimator $\hat{e}\left(\mathbf{u}_{h}, \hat{\boldsymbol{\sigma}}_{h}\right)$ and of the global lower bound $\tilde{e}\left(\tilde{\mathbf{u}}_{h}-\mathbf{u}_{h}, \hat{\boldsymbol{\sigma}}_{h}-\boldsymbol{\sigma}_{h}\right)$ is estimated by an effectivity index:

$$
\eta_{u}=\frac{\hat{e}\left(\mathbf{u}_{h}, \hat{\boldsymbol{\sigma}}_{h}\right)}{e_{\mathrm{ref}}} \quad \text { and } \quad \eta_{L}=\frac{\tilde{e}\left(\tilde{\mathbf{u}}_{h}-\mathbf{u}_{h}, \hat{\boldsymbol{\sigma}}_{h}-\boldsymbol{\sigma}_{h}\right)}{e_{\mathrm{ref}}}
$$

where $e_{\text {ref }}=e_{h}$ when the exact error is available and $e_{\text {ref }}$ is computed from a refined mesh when the exact error is not available. 


\subsection{Behavior of the global energy error}

In this section, the study of the effectivity indexes and the convergence rate of the global errors estimators $\tilde{e}$ and $\hat{e}$ shows the quality of the global lower and upper bounds presented in Sections 2 and 4. Two structures are studied. The first structure is a thin elastic plate with two holes proposed in [30], this is an in-plane stress linear elastic problem loaded with an unit tension along the vertical edges (see Figure 2). The symmetry of the problem allows us to study only one-fourth of the plate. The structure is meshed with 6-nodes triangular elements (as shown in Figure 3). The second structure is a holed plate submitted to bending. The plate clamped on the left edge and loaded with an uniform load is depicted in Figure 4. The initial coarse mesh (6-nodes triangular elements) is shown in Figure 5. The material properties are $E=100 \mathrm{GPa}$ and $v=0.3$. Owing to the lack of analytical solution for these problems, we use a reference solution computed on a fine mesh. The reference solution is then used to compute the reference error $e_{\text {ref }}$. The behavior of the

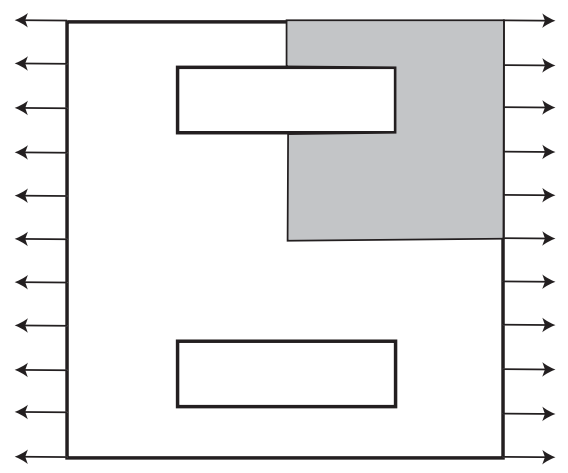

Figure 2. Model problem of the elastic plate with two holes.

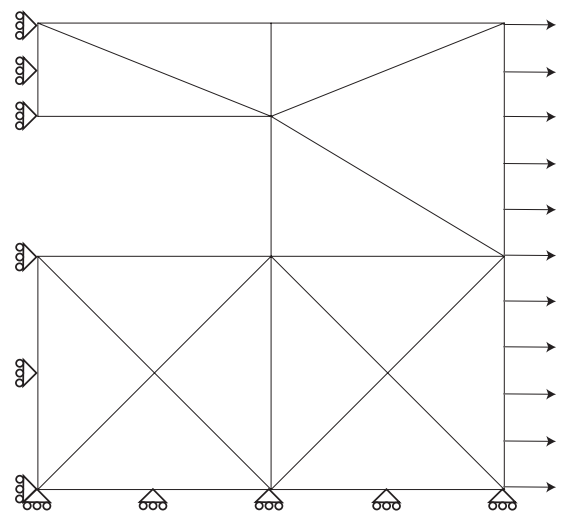

Figure 3. Plate with two holes: one-fourth of the plate is meshed. 


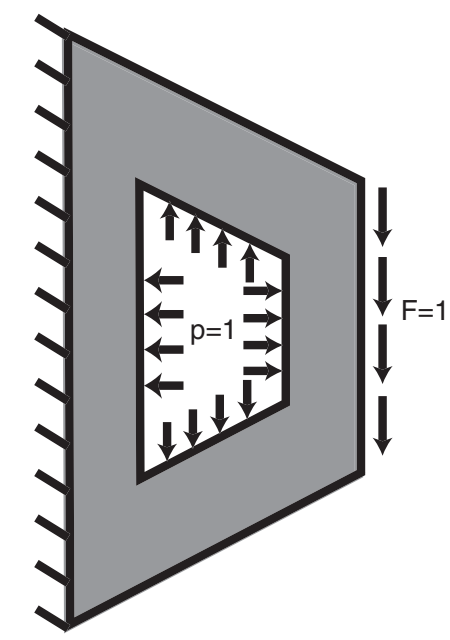

Figure 4. Model problem of the elastic plate submitted to bending.

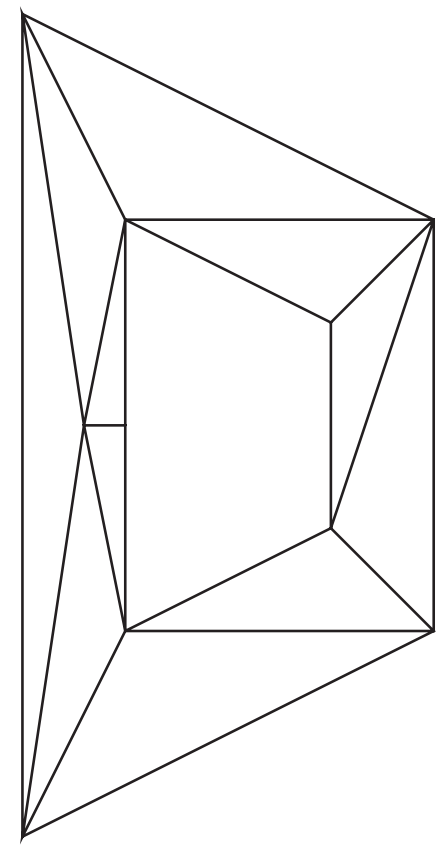

Figure 5. Elastic plate submitted to bending: coarse mesh.

global CRE estimator (the upper bound) and global lower bound is analyzed by refining the initial mesh. The local problems (25) defined on $\omega_{i}$ are solved on a subdivision of the initial FE mesh $P_{h, 3}\left(\omega_{i}\right)$. 


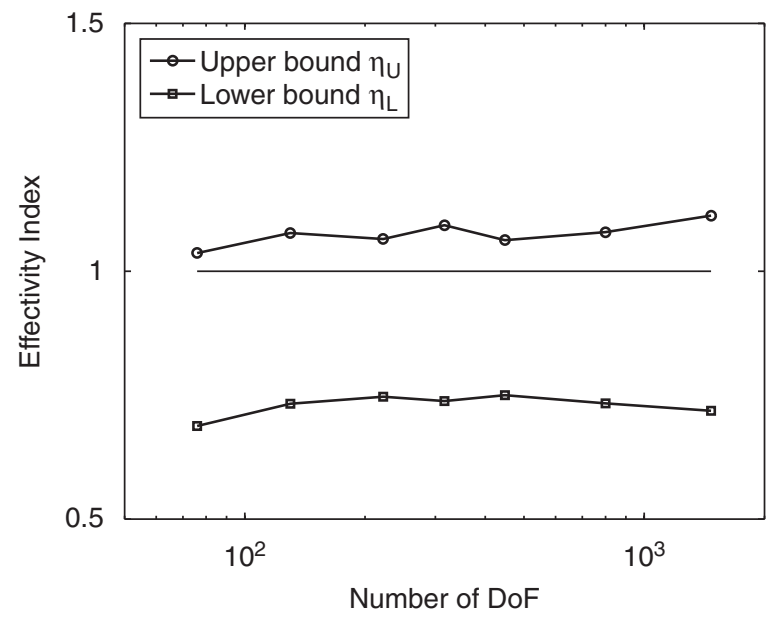

Figure 6. Plate with two holes: upper and lower bound as a function of DoF.

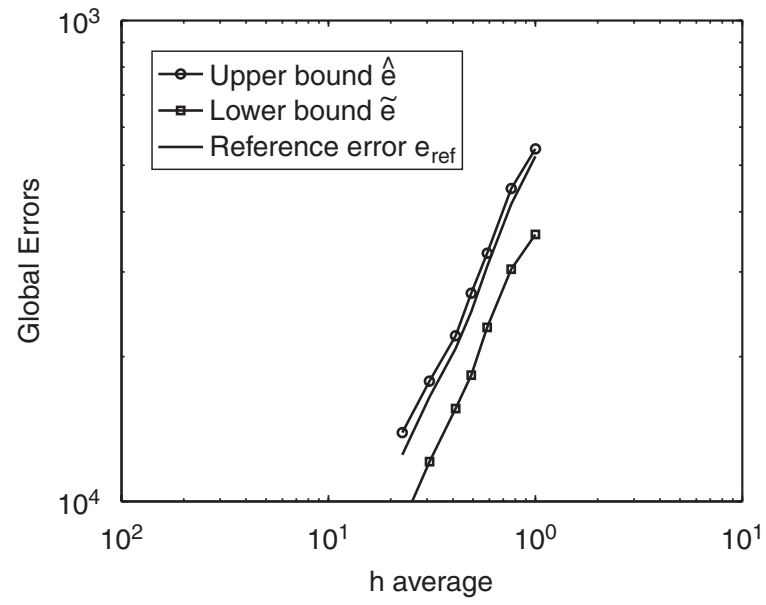

Figure 7. Plate with two holes: convergence rates for the upper bound, the lower bound and the reference error.

Figure 6 presents the evolution of the effectivity indexes for the CRE estimator and for the global lower bound. In this example, the mean effectivity index of the CRE estimator is $\eta_{\mathrm{U}}=1.08$. The mean effectivity index of the lower bound is not as sharp: $\eta_{\mathrm{L}}=0.73$. Figure 7 presents the convergence rates of $\hat{e}, e_{\text {ref }}$ and $\tilde{e}$. The three quantities have similar convergence rates, which are equal to 1 . We do not obtain the theoretical convergence rate $p=2$ of bilinear elements because of the presence of the corner singularities. Table I presents the numerical results for the second example, a function of the number of dof: the relative error estimator $\hat{\varepsilon}$, the global upper bound $\hat{e}$, the global lower bound $\tilde{e}$, the reference error $e_{\text {ref }}$, the effectivity indices $\eta_{\mathrm{L}}$ and $\eta_{\mathrm{U}}$. The evolutions of the effectivity indices are represented in Figure 8. The results obtained for this problem are 
Table I. Elastic plate submitted to bending.

\begin{tabular}{|c|c|c|c|c|c|c|}
\hline Number of Dof & 62 & 122 & 224 & 476 & 784 & 1572 \\
\hline$\hat{\varepsilon}(\%)$ & 43.03 & 21.27 & 15.48 & 11.92 & 8.618 & 6.887 \\
\hline$\hat{e}$ & 0.3708 & 0.1988 & 0.1490 & 0.1165 & $8.4690 \mathrm{E}-02$ & $6.7958 \mathrm{E}-02$ \\
\hline$\tilde{e}$ & 0.1271 & 0.1241 & $9.8705 \mathrm{E}-02$ & $7.1684 \mathrm{E}-02$ & $5.7886 \mathrm{E}-02$ & $4.4531 \mathrm{E}-02$ \\
\hline$e_{\text {ref }}$ & 0.2952 & 0.1881 & 0.1375 & 0.1010 & $7.9173 \mathrm{E}-02$ & $6.2185 \mathrm{E}-02$ \\
\hline$\eta_{\mathrm{U}}$ & 1.256 & 1.056 & 1.083 & 1.153 & 1.069 & 1.092 \\
\hline$\eta_{\mathrm{L}}$ & 0.430 & 0.659 & 0.717 & 0.709 & 0.731 & 0.716 \\
\hline
\end{tabular}

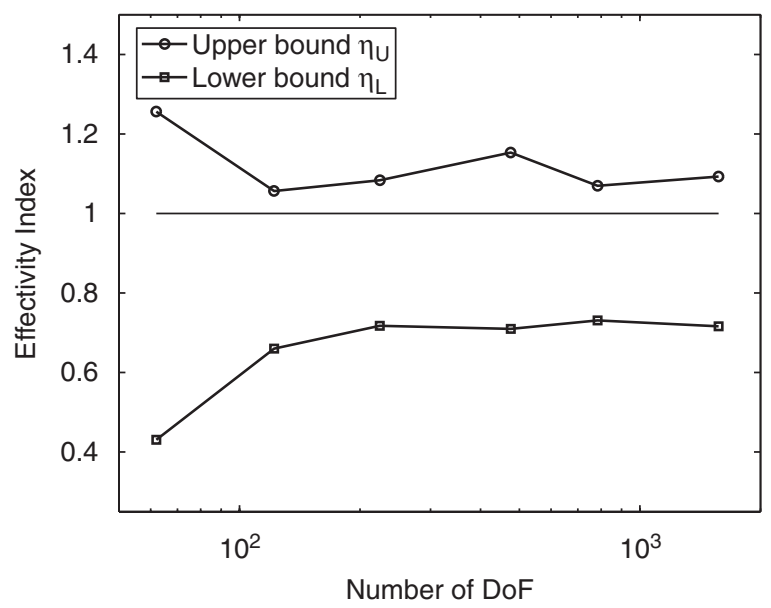

Figure 8. Elastic plate submitted to bending: upper and lower bound as a function of DoF.

similar to those obtained in the first example, with an upper bound sharper than the lower bound (mean effectivity indexe: $\eta_{\mathrm{U}}=1.11$ for the upper bound, $\eta_{\mathrm{L}}=0.66$ for the lower bound).

\subsection{Application to goal-oriented error estimation in a crack opening problem}

This section illustrates the behavior of the goal-oriented error estimator presented in Section 5 compared with the simpler version proposed in [12]. The first example shows that for a simple tension state we obtain an improvement of the lower bound, but not for the upper bound. The second example illustrates that for a more complex state of tension, the upper bound is also greatly improved.

For a crack opening problem, a classical quantity of interest is the mean vertical displacement along the crack boundaries

$$
I=\frac{1}{a}\left(\int_{\Gamma_{1}} \mathbf{u} \cdot(-\mathbf{y}) \mathrm{d} \Gamma+\int_{\Gamma_{2}} \mathbf{u} \cdot(\mathbf{y}) \mathrm{d} \Gamma\right)
$$

where $a$ is the crack length and $\mathbf{y}$ is the normal unit vector along the crack edge. 


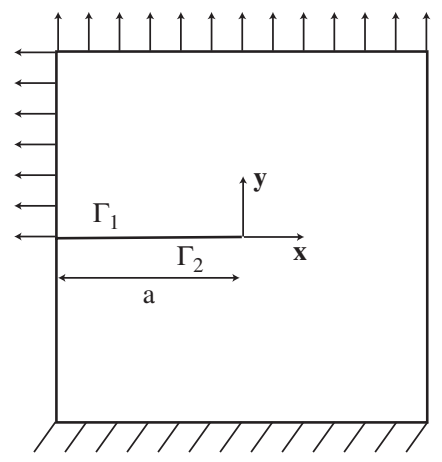

Figure 9. Horizontal crack opening problem.

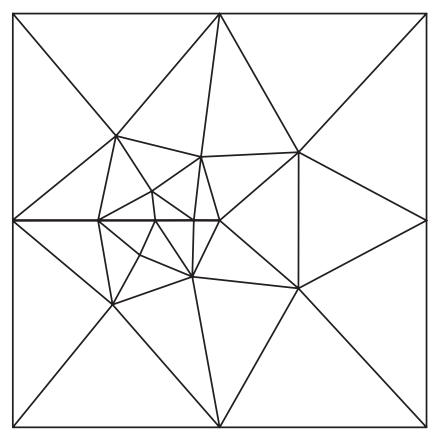

Figure 10. Horizontal crack opening problem: coarsest mesh.

The computed quantity is:

$$
I_{h}=\frac{1}{a}\left(\int_{\Gamma_{1}} \mathbf{u}_{h} \cdot(-\mathbf{y}) \mathrm{d} \Gamma+\int_{\Gamma_{2}} \mathbf{u}_{h} \cdot(\mathbf{y}) \mathrm{d} \Gamma\right)
$$

The material properties are $E=100 \mathrm{GPa}$ and $v=0.3$. The structure is studied in plane stress and meshed with 6-nodes triangular elements. The reference solution $\mathbf{u}_{\mathrm{ref}}$ is computed on a very refined mesh. A reference quantity of interest $I_{\text {ref }}$ is computed by replacing $\mathbf{u}$ by $\mathbf{u}_{\text {ref }}$ in Equation (38). The local problems (25) defined on $\omega_{i}$ are solved on a subdivision of the initial FE mesh $P_{h, 3}\left(\omega_{i}\right)$.

The first example is a thin plate with a single edge horizontal crack subjected to a tension state represented in Figure 9. Four meshes are considered; the coarsest mesh is represented in Figure 10. On each mesh, the quantities $I_{h}, \eta_{\text {upp }}$ and $\eta_{\text {low }}$ as well as $\eta_{\text {low }}^{\mathrm{CRE}}$ and $\eta_{\text {upp }}^{\mathrm{CRE}}$ have been computed and are reported in Table II.

A first observation is that the lower bound $\eta_{\text {low }}$ for $I-I_{h}$ is greater than zero. This means that the lower bound for the quantity of interest $I_{h}+\eta_{\text {low }}$ is greater than the computed quantity of interest $I_{h}$. The second observation is that the upper bound obtained directly with the CRE 
Table II. Horizontal crack opening problem: upper and lower bounds for $I-I_{h}$.

\begin{tabular}{lcccc}
\hline Number of Dof & 154 & 316 & 590 & 1168 \\
\hline$I_{h}$ & $2.9816 \mathrm{E}-05$ & $3.2939 \mathrm{E}-05$ & $3.4195 \mathrm{E}-05$ & $3.4771 \mathrm{E}-05$ \\
$\eta_{\text {low }}$ & $2.8459 \mathrm{E}-06$ & $1.3049 \mathrm{E}-06$ & $6.8234 \mathrm{E}-07$ & $3.8664 \mathrm{E}-07$ \\
$\eta_{\text {upp }}$ & $6.1811 \mathrm{E}-06$ & $3.1367 \mathrm{E}-06$ & $1.7428 \mathrm{E}-06$ & $1.1824 \mathrm{E}-06$ \\
$\eta_{\text {low }}^{\text {lRE }}$ & $-4.048 \mathrm{E}-06$ & $-2.004 \mathrm{E}-06$ & $-1.108 \mathrm{E}-06$ & $-0.731 \mathrm{E}-06$ \\
$\eta_{\text {upp }}^{\text {CRE }}$ & $6.398 \mathrm{E}-06$ & $3.236 \mathrm{E}-06$ & $1.796 \mathrm{E}-06$ & $1.215 \mathrm{E}-06$ \\
\hline
\end{tabular}

Table III. Horizontal crack opening problem: upper and lower bounds for the quantity of interest.

\begin{tabular}{|c|c|c|c|c|}
\hline Number of Dof & 154 & 316 & 590 & 1168 \\
\hline$I_{h}^{\mathrm{upp}}-I_{h}^{\text {low }}$ & $3.34 \mathrm{E}-06$ & $1.83 \mathrm{E}-06$ & $1.06 \mathrm{E}-06$ & $7.96 \mathrm{E}-07$ \\
\hline$I_{h}^{\text {upp,CRE }}-I_{h}^{\text {low,CRE }}$ & $6.80 \mathrm{E}-06$ & $3.44 \mathrm{E}-06$ & $1.91 \mathrm{E}-06$ & $1.29 \mathrm{E}-06$ \\
\hline$\frac{I_{h}^{\mathrm{upp}, \mathrm{CRE}}-I_{h}^{\text {low,CRE }}}{I_{h}^{\mathrm{upp}}-I_{h}^{\text {low }}}$ & 2.04 & 1.88 & 1.80 & 1.62 \\
\hline
\end{tabular}

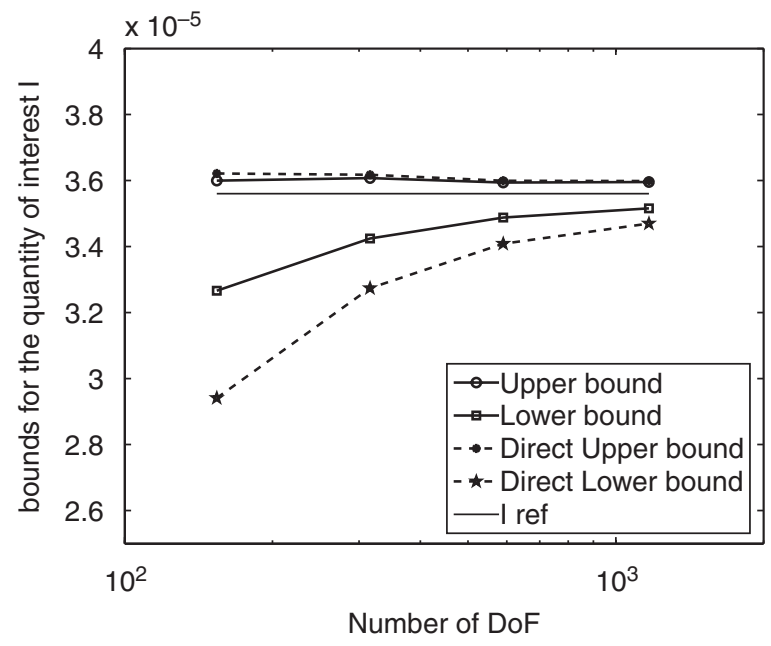

Figure 11. Horizontal crack opening problem: upper and lower bounds for $I$.

estimator $\eta_{\text {upp }}^{\mathrm{CRE}}$ is close to the improved local upper bound $\eta_{\text {upp }}$ defined in Section 5 . However, if the lower bound is considered then the introduction of the improved lower bound $\eta_{\text {low }}$ leads to really sharper bounds compared with $\eta_{\mathrm{low}}^{\mathrm{CRE}}$ (see Table II). This improvement is illustrated in Table III, where we have computed the size of the intervals $\left(I_{h}^{\mathrm{upp}, \mathrm{CRE}}-I_{h}^{\mathrm{low}, \mathrm{CRE}}\right)$ and $\left(I_{h}^{\mathrm{upp}}-I_{h}^{\mathrm{low}}\right)$ 


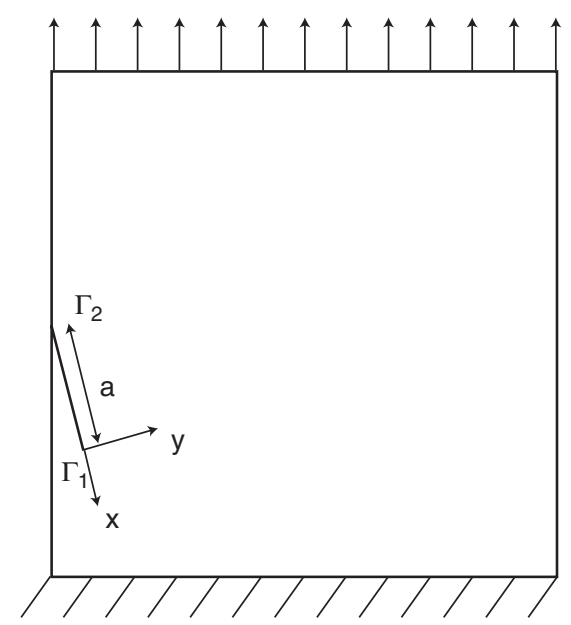

Figure 12. Oblique crack opening problem.

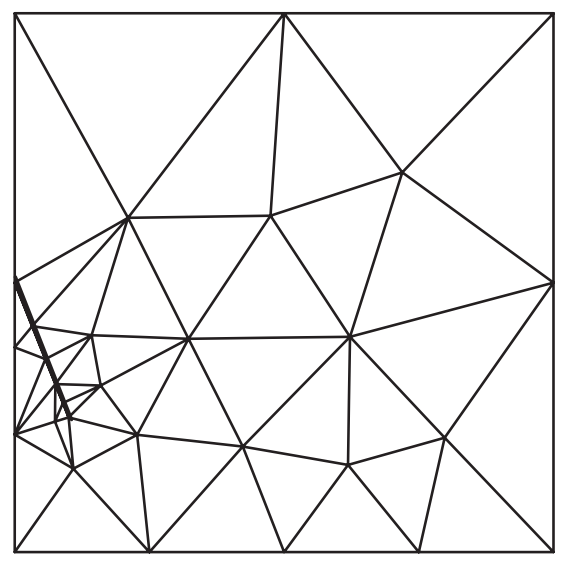

Figure 13. Oblique crack opening problem: coarsest mesh.

and their ratio. In Figure 11 we have represented the evolution of the bounds for the quantity of interest as a function of the number of DoF.

The second example is a thin plate with a single edge oblique crack subjected to a tension state represented in Figure 12. Four meshes are considered, the coarsest mesh is represented in Figure 13. On each mesh the quantities $I_{h}, \eta_{\text {upp }}$ and $\eta_{\text {low }}$ as well as $\eta_{\text {low }}^{\text {CRE }}$ and $\eta_{\text {upp }}^{\text {CRE have been }}$ computed and are reported in Table IV. Both the upper bound $\eta_{\text {upp }}$ and the lower bound $\eta_{\text {low }}$ are sharper than $\eta_{\text {upp }}^{\mathrm{CRE}}$ and $\eta_{\mathrm{low}}^{\mathrm{CRE}}$. The range obtained for the quantity of interest is divided by a 
Table IV. Oblique crack opening problem: upper and lower bounds for $I-I_{h}$.

\begin{tabular}{lrrrr}
\hline Number of Dof & \multicolumn{1}{c}{230} & \multicolumn{1}{c}{580} & \multicolumn{1}{c}{1152} & \multicolumn{1}{c}{2048} \\
\hline$I_{h}$ & $0.1461 \mathrm{E}-05$ & $0.1493 \mathrm{E}-05$ & $0.1541 \mathrm{E}-05$ & $0.1564 \mathrm{E}-05$ \\
$\eta_{\text {low }}$ & $-0.0708 \mathrm{E}-06$ & $-0.1162 \mathrm{E}-06$ & $-0.0391 \mathrm{E}-06$ & $-0.0259 \mathrm{E}-06$ \\
$\eta_{\text {upp }}$ & $0.5977 \mathrm{E}-06$ & $0.3164 \mathrm{E}-06$ & $0.1755 \mathrm{E}-06$ & $0.0907 \mathrm{E}-06$ \\
$\eta_{\text {low }}^{\text {CRE }}$ & $-0.5220 \mathrm{E}-06$ & $-0.4589 \mathrm{E}-06$ & $-0.1922 \mathrm{E}-06$ & $-0.0956 \mathrm{E}-06$ \\
$\eta_{\text {upp }}^{\text {CRE }}$ & $0.8387 \mathrm{E}-06$ & $0.5510 \mathrm{E}-06$ & $0.2779 \mathrm{E}-06$ & $0.1385 \mathrm{E}-06$ \\
$\frac{I_{h}^{\text {upp }, \mathrm{CRE}}-I_{h}^{\text {low }, \mathrm{CRE}}}{\frac{I_{h}^{\text {upp }}-I_{h}^{\text {low }}}{2}}$ & 2.04 & 2.33 & 2.19 & 2.01 \\
\hline
\end{tabular}

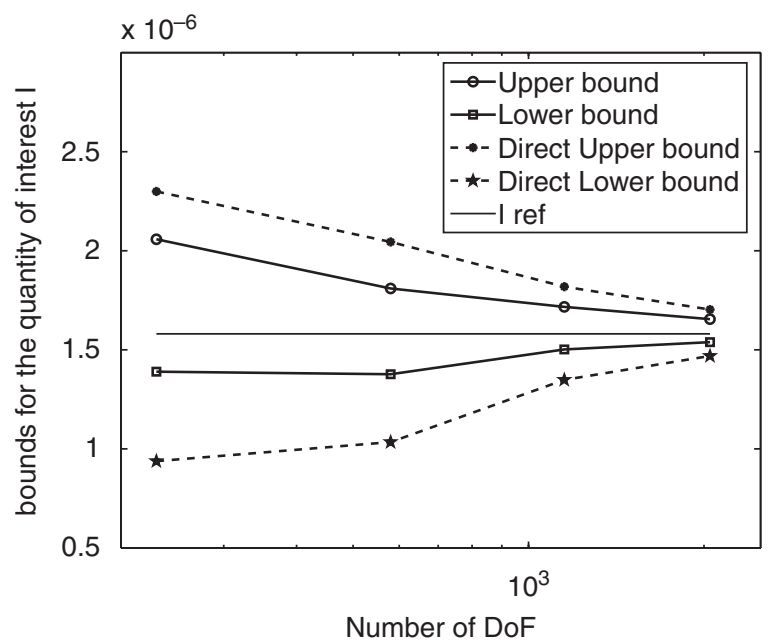

Figure 14. Oblique cracked plate problem: upper and lower bounds for $I$.

factor 2 as shown in Table IV. In Figure 14 we have represented the evolution of the bounds for the quantity of interest as a function of the number of DoF.

\section{CONCLUSION}

This paper has introduced a construction for the admissible stress fields used in the CRE estimator, which is more simple than the classical techniques proposed in previous papers. This technique leads to very good effectivity indexes. Based on these admissible stress fields, a lower bound for the energy error is defined. This lower bound can be combined with the CRE estimator (which is an upper bound for the energy error) so as to obtain lower and upper bounds for quantities of interest by a classical approach. This combined approach allows to obtain sharper bounds for a quantity of interest in comparison with alternative works based only on the constitutive relation approach. 


\section{APPENDIX A}

When $\lambda_{i} \mathbf{u}_{\mathrm{rb}}$ does not belong to $\mathscr{U}^{h}$ it is necessary to add forces $\mathbf{F}_{i}$ to the loading on each domain $\omega_{i}$ such that the resultant and the moment vanish on that domain and such that the sum of $\mathbf{F}_{i}$ over the whole structure is zero. To simplify the presentation, we will limit ourselves to the case where $\mathbf{T}$ is equal to zero on $\partial_{2} \Omega$. The forces are added on the vertices of the initial mesh $\mathscr{P}_{h}$. Each vertex $i \in \mathbf{I}_{\mathrm{v}}$ is associated with a domain $\omega_{i}$, the position of the vertex $j$ is denoted by $M_{j}$. In a domain $\omega_{i}$, the force added on the vertex $j$ is defined by

$$
\mathbf{g}_{i j}=\int_{\omega_{i}}\left(\boldsymbol{\sigma}_{h}\left(\lambda_{j} \operatorname{grad} \lambda_{i}+\lambda_{i} \operatorname{grad} \lambda_{j}\right)-\lambda_{i} \lambda_{j} \mathbf{b}\right) \mathrm{d} \Omega
$$

Let us denote the set of vertices of the FE mesh belonging to $\omega_{i}$ by $\mathbf{I}_{\mathrm{v}}\left(\omega_{i}\right)$. Equation (22) is modified and the admissible stress $\hat{\boldsymbol{\sigma}}_{h}^{i}$ in $\omega_{i}$ is sought as a solution of the following modified problem:

$$
\int_{\omega_{i}} \hat{\boldsymbol{\sigma}}_{h}^{i} \varepsilon\left(\mathbf{u}^{*}\right) \mathrm{d} \omega=\mathscr{R}^{\bmod }\left(\mathbf{u}^{*}\right) \quad \forall \mathbf{u}^{*} \in \mathscr{U}_{0}\left(\omega_{i}\right)
$$

with

$$
\mathscr{R}^{\bmod }\left(\mathbf{u}^{*}\right)=\int_{\omega_{i}}\left(\lambda_{i} \mathbf{b}-\boldsymbol{\sigma}_{h} \operatorname{grad} \lambda_{i}\right) \mathbf{u}^{*} \mathrm{~d} \omega+\sum_{j \in \mathbf{I}_{\mathbf{v}}\left(\omega_{i}\right)} \mathbf{g}_{i j} \mathbf{u}_{j}^{*}
$$

where $\mathbf{u}_{j}^{*}=\mathbf{u}^{*}\left(M_{j}\right)$.

Proposition A.1

The nodal forces $\mathbf{g}_{i j}$ do not modify the global equilibrium on the structure $\Omega$ (i.e. $\sum_{i \in \mathbf{I}_{v}} \mathbf{g}_{i j}=0$ ).

Proof

Let us consider the sum of the nodal forces over the set of the vertices $\mathbf{I}_{\mathrm{v}}$. As $\lambda_{i}=0$ in $\Omega-\omega_{i}$ we have the following relations:

$$
\begin{aligned}
\sum_{i \in \mathbf{I}_{\mathrm{v}}} \mathbf{g}_{i j} & =\sum_{i \in \mathbf{I}_{\mathrm{v}}}\left(\int_{\omega_{i}}\left(\boldsymbol{\sigma}_{h}\left(\lambda_{j} \operatorname{grad} \lambda_{i}+\lambda_{i} \operatorname{grad} \lambda_{j}\right)-\lambda_{i} \lambda_{j} \mathbf{b}\right) \mathrm{d} \omega\right) \\
& =\sum_{i \in \mathbf{I}_{\mathrm{v}}}\left(\int_{\Omega}\left(\boldsymbol{\sigma}_{h}\left(\lambda_{j} \operatorname{grad} \lambda_{i}+\lambda_{i} \operatorname{grad} \lambda_{j}\right)-\lambda_{i} \lambda_{j} \mathbf{b}\right) \mathrm{d} \Omega\right) \\
& =\int_{\Omega}\left(\boldsymbol{\sigma}_{h}\left(\lambda_{j} \operatorname{grad}\left(\sum_{i \in \mathbf{I}_{\mathrm{v}}} \lambda_{i}\right)+\left(\sum_{i \in \mathbf{I}_{\mathrm{v}}} \lambda_{i}\right) \operatorname{grad} \lambda_{j}\right)-\left(\sum_{i \in \mathbf{I}_{\mathrm{v}}} \lambda_{i}\right) \lambda_{j} \mathbf{b}\right) \mathrm{d} \Omega
\end{aligned}
$$

The set $\left(\lambda_{i}\right)_{i \in \mathbf{I}_{\mathrm{v}}}$ is a partition of unity: $\sum_{i \in \mathbf{I}_{\mathrm{v}}} \lambda_{i}=1$ and $\operatorname{grad}\left(\sum_{i \in \mathbf{I}_{\mathrm{v}}} \lambda_{i}\right)=0$. The global force applied to the vertex $j$ is:

$$
\begin{aligned}
\sum_{i \in \mathbf{I}_{\mathrm{v}}} \mathbf{g}_{i j} & =\int_{\Omega}\left(\boldsymbol{\sigma}_{h} \operatorname{grad} \lambda_{j}-\lambda_{j} \mathbf{b}\right) \mathrm{d} \Omega \\
& =0\left(\boldsymbol{\sigma}_{h} \text { because it is in equilibrium in the FE sense }\right)
\end{aligned}
$$


Proposition A.2

$\mathscr{R}^{\mathrm{mod}}\left(\mathbf{u}_{\mathrm{rb}}\right)=0$ for any rigid body motion of $\omega_{i}$.

Proof

On a domain $\omega_{i}$ the rigid body motion is written on the basis of the linear shape functions

$$
\mathbf{u}_{\mathrm{rb}}=\sum_{j \in \mathbf{I}_{\mathrm{v}}\left(\omega_{i}\right)} \lambda_{j} \mathbf{q}_{\mathrm{rb}}^{j}
$$

where $\mathbf{q}_{\mathrm{rb}}^{j}$ is the displacement of the vertice $j$

$$
\mathbf{R}^{\bmod }\left(\mathbf{u}_{\mathrm{rb}}\right)=\int_{\omega_{i}}\left(\lambda_{i} \mathbf{b}-\boldsymbol{\sigma}_{h} \operatorname{grad} \lambda_{i}\right) \mathbf{u}_{\mathrm{rb}} \mathrm{d} \omega+\sum_{j \in \mathbf{I}_{\mathrm{v}}\left(\omega_{i}\right)} \mathbf{g}_{i j} \mathbf{q}_{\mathrm{rb}}^{j}
$$

and

$$
\begin{aligned}
\sum_{j \in \mathbf{I}_{\mathrm{v}}\left(\omega_{i}\right)} \mathbf{g}_{i j} \mathbf{q}_{\mathrm{rb}}^{j}= & \sum_{j \in \mathbf{I}_{\mathrm{v}}\left(\omega_{i}\right)}\left(\int_{\omega_{i}}\left(\boldsymbol{\sigma}_{h}\left(\lambda_{j} \operatorname{grad} \lambda_{i}+\lambda_{i} \operatorname{grad} \lambda_{j}\right)-\lambda_{i} \lambda_{j} \mathbf{b}\right) \mathrm{d} \omega\right) \cdot \mathbf{q}_{\mathrm{rb}}^{j} \\
= & \int_{\omega_{i}}\left(\sum_{j \in \mathbf{I}_{\mathrm{v}}\left(\omega_{i}\right)} \lambda_{j} \mathbf{q}_{\mathrm{rb}}^{j}\right)\left(\boldsymbol{\sigma}_{h} \operatorname{grad} \lambda_{i}-\lambda_{i} \mathbf{b}\right) \mathrm{d} \omega \\
& +\int_{\omega_{i}} \lambda_{i} \boldsymbol{\sigma}_{h}\left(\sum_{j \in \mathbf{I}_{\mathrm{v}}\left(\omega_{i}\right)} \operatorname{grad} \lambda_{j} \mathbf{q}_{\mathrm{rb}}^{j}\right) \mathrm{d} \omega \\
= & \int_{\omega_{i}} \mathbf{u}_{\mathrm{rb}} \cdot\left(\boldsymbol{\sigma}_{h} \operatorname{grad} \lambda_{i}-\lambda_{i} \mathbf{b}\right)+\lambda_{i} \boldsymbol{\sigma}_{h}\left(\operatorname{grad} \mathbf{u}_{\mathrm{rb}}\right) \mathrm{d} \omega
\end{aligned}
$$

as $\mathbf{u}_{\mathrm{rb}}$ is a rigid body motion $\operatorname{grad} \mathbf{u}_{\mathrm{rb}}=0$ and $\mathscr{R}^{\bmod }\left(\mathbf{u}_{\mathrm{rb}}\right)=\int_{\omega_{i}}\left(\lambda_{i} \mathbf{b}-\boldsymbol{\sigma}_{h} \operatorname{grad} \lambda_{i}\right) \mathbf{u}_{\mathrm{rb}} \mathrm{d} \omega+\int_{\omega_{i}} \mathbf{u}_{\mathrm{rb}}$. $\left(\boldsymbol{\sigma}_{h} \operatorname{grad} \lambda_{i}-\lambda_{i} \mathbf{b}\right) \mathrm{d} \omega=0$.

\section{REFERENCES}

1. Babuška I, Rheinboldt WC. A posteriori estimates for the finite element method. International Journal for Numerical Methods in Engineering 1978; 12:1597-1615.

2. Ladevèze P, Leguillon D. Error estimate procedure in the finite element method and application. SIAM Journal on Numerical Analysis 1983; 20(3):485-509.

3. Zienkiewicz OC, Zhu JZ. A simple error estimator and adaptive procedure for practical engineering analysis. International Journal for Numerical Methods in Engineering 1987; 24:337-357.

4. Babuška I, Miller A. The post-processing approach of the finite element method. Part II: the calculation of the stress intensity factor. International Journal for Numerical Methods in Engineering 1982; 20:1111-1129.

5. Kelly DW, Isles JD. Procedures for residual equilibration and local error estimation in the finite element method. Communications in Applied Numerical Methods 1989; 5:497-505.

6. Rannacher R, Stuttmeier FT. A feedback approach to error control in finite element methods: application to linear elasticity. Computational Mechanics 1997; 19:434-446.

7. Peraire J, Patera A. Bounds for linear-functional outputs of coercive partial differential equations: local indicators and adaptive refinement. In Advances in Adaptive Computational Methods, Ladevèze P, Oden JT (eds). Elsevier: Amsterdam, 1998; 199-216.

8. Prudhomme S, Oden JT. On goal-oriented error estimation for elliptic problems: application to the control of pointwise errors. Computer Methods in Applied Mechanics and Engineering 1999; 176:313-331. 
9. Ladevèze P, Rougeot P, Blanchard P, Moreau JP. Local error estimators for finite element analysis. Computer Methods in Applied Mechanics and Engineering 1999; 176:231-246.

10. Ohnimus S, Stein E, Walhorn E. Local error estimates of fem for displacements and stresses in linear elasticity by solving local Neumann problems. International Journal for Numerical Methods in Engineering 2001; 52:727-746.

11. Prudhomme S, Oden JT, Westermann T, Bass J, Botkin ME. Practical methods for a posteriori error estimation in engineering applications. International Journal for Numerical Methods in Engineering 2003; 56:1193-1224.

12. Gallimard L, Panetier J. Error estimation of stress intensity factors for mixed-mode cracks. International Journal for Numerical Methods in Engineering 2006; 68(3):299-316.

13. Ladevèze P, Pelle JP, Rougeot P. Error estimation and mesh optimization for classical finite elements. Engineering Computations 1991; 8:69-80.

14. Machiels L, Maday Y, Patera AT. A 'flux-free' nodal Neumann subproblem approach to output bounds for partial differential equations. Comptes Rendus de l'Académie des Sciences, Série I 2000; 330(3):249-254.

15. Ainsworth M, Oden TJ. A Posteriori Error Estimation in Finite Element Analysis. Wiley: Chichester, 2000.

16. Luce R, Wohlmuth B. A local a posteriori error estimator based on equilibrated fluxes. SIAM Journal on Numerical Analysis 2004; 42:1394-1414.

17. Parés N, Dìez P, Huerta A. Subdomain-based flux-free a posteriori error estimators. Computer Methods in Applied Mechanics and Engineering 2006; 195(4-6):297-323.

18. Nicaise S, Witowski K, Wohlmuth BI. An a posteriori error estimator for the Lamé equation based on $H$ (div)conforming stress approximations. IMA Journal for Numerical Analysis 2008; 28(2):331-353.

19. Gallimard L, Ladevèze P, Pelle JP. An enhanced error estimator on constitutive relation for plasticity problems. Computers and Structures 2000; 78:801-810.

20. Florentin E, Gallimard L, Pelle JP. Evaluation of the local quality of stresses in 3d finite element analysis. Computer Methods in Applied Mechanics and Engineering 2002; 191(18-19):1751-1757.

21. Prager W, Synge JL. Approximation in elasticity based on the concept of functions space. Quarterly of Applied Mathematics 1947; 5:261-269.

22. Strouboulis T, Babuška I. The Finite Element Methods and Its Reliability. Clarendon Press: Oxford, 2001.

23. Morin P, Nochetto RH, Siebert KG. Local problems on stars: a posteriori error estimators, convergence and performance. Mathematics of Computation 2003; 72(243):1067-1097.

24. Choi HW, Paraschivoiu M. Adaptive computations of a posteriori finite element output bounds: a comparison of the 'hybrid-flux' approach and the 'flux-free' approach. Computer Methods in Applied Mechanics and Engineering 2004; 193(36-38):4001-4033.

25. Strouboulis T, Babuška I, Datta DK, Copps K, Gangaraj SK. A posteriori estimation and adaptative control of the error in the quantity of interest. Part 1: a posteriori estimation of the error in the von Mises stress and the stress intensity factor. Computer Methods in Applied Mechanics and Engineering 2000; 180:261-274.

26. Beckers R, Rannacher R. An optimal control approach to a posteriori error estimation in finite element method. Acta Numerica 2001; 19:1-102.

27. Heintz P, Samuelsson K. On adaptive strategies and error control in fracture mechanics. Computers and Structures 2004; 82:485-497.

28. Ruter M, Stein E. Goal-oriented a posteriori error in linear elastic fracture mechanics. Computer Methods in Applied Mechanics and Engineering 2006; 195:251-278.

29. Gallimard L. Evaluation of the local quality of the von mises's stress and 12-norm of the stress. Engineering Computations 2006; 23(7-8):876-897.

30. Peraire J, Patera AT. Bounds for linear-functional outputs of coercive partial differential equations: local indicators and adaptive refinement. Advances in Adaptive Computational Methods in Mechanics (Cachan, 1997). Studies in Applied Mechanics, vol. 47. Elsevier: Amsterdam, 1998; 199-216. 